\title{
Pneumothorax as the presenting manifestation of COVID-19
}

\author{
Shahin Ayazi ${ }^{1}$, Javad Zebarjadi $^{2}$, Andrew D. Grubic ${ }^{1}$, Hamed Tahmasbi ${ }^{2}$, Khosro Ayazi ${ }^{2}$, Blair A. Jobe ${ }^{1}$ \\ ${ }^{1}$ Esophageal and Lung Institute, Department of Surgery, Allegheny Health Network, Pittsburgh, PA, USA; ${ }^{2}$ Department of Surgery, Imam Hossein \\ Hospital, Shahid Beheshti University of Medical Sciences, Tehran, Iran \\ Correspondence to: Shahin Ayazi, MD. Esophageal and Lung Institute, Department of Surgery, Allegheny Health Network, 4815 Liberty Avenue, Suite \\ 439, Pittsburgh, PA 15224, USA. Email: shahin.ayazi@ahn.org.
}

Submitted Apr 17, 2020. Accepted for publication Aug 28, 2020.

doi: $10.21037 /$ jtd-20-1687

View this article at: http://dx.doi.org/10.21037/jtd-20-1687

Since identification of novel severe acute respiratory syndrome coronavirus 2 (SARS-CoV-2) as the causative agent from a cluster of pneumonias in the Hubei providence of China in December 2019, coronavirus disease 2019 (COVID-19) has rapidly evolved into a global pandemic. Iran has been one the countries most affected by COVID-19, with a mortality rate of $7.6 \%$ among 29,406 confirmed cases as of March 27th, 2020 (1). COVID-19 was first reported in 2 patients in the city of Qom on February 19th, 2020, since then the disease has disseminated throughout all 31 provinces. The first confirmed patients in the capital city of Tehran were reported on February 21st 2020 (2). In this manuscript, we report three COVID-19 patients from Tehran who presented with pneumothorax as an initial manifestation of COVID-19. This unusual clinical presentation has not been previously reported, and its addition to the rapidly growing list of signs and symptoms could increase awareness to this potentially life-threatening consequence of COVID-19.

All 3 patients presented to the emergency room of Imam Hossein Hospital (Table 1), a University hospital located in Southeast of Tehran, Iran. The clinical presentation of these patients raised concern for COVID-19 and this prompted evaluation by the emergency room (ER) physicians. Chest computerized tomography (CT) scan revealed a large pneumothorax in all 3 patients and the surgical service was then consulted to assist with management.
Patient specific clinical course and pertinent clinical and laboratory findings

\section{Patient 1}

On March 20, 2020, a 45 -year-old homeless man presented to the emergency department with a 5-day course of fever, cough, and shortness of breath. He was non-smoker and had no known previous past medical or surgical history and denied any recent trauma. The physical examination on arrival revealed a body temperature of $38^{\circ} \mathrm{C}$ with blood pressure of $110 / 80 \mathrm{mmHg}$, pulse rate of 105 beats per minute and respiratory rate of 28 breaths per minute, his oxygen saturation was $89 \%$ on room air. He had no alteration in mental status with a Glasgow coma scale (GCS) of 15. Lung auscultation revealed rhonchi on the right side and diminished breath sounds over the left hemithorax. Initial blood work demonstrated the following: WBC 4,600 $\mu \mathrm{L}$ with $17 \%$ lymphocytes, CRP $51 \mathrm{mg} / \mathrm{L}$, with a non-elevated D-Dimer test $(<7,500 \mathrm{ng} / \mathrm{mL})$. Sample was obtained and sent for real time reverse transcriptionpolymerase chain reaction (RT-PCR) analysis to evaluate for COVID-19. Chest CT scan showed a large left sided pneumothorax (Figure 1). A left sided thoracostomy tube was placed and the lung completely re-expanded. Despite this measure, his respiratory status continued to deteriorate, and he was transferred to the intensive care unit (ICU), and required intubation and ventilator support 8 hours after placement of the thoracostomy tube. On arrival to the ICU,
^ ORCID: 0000-0002-8113-9164. 2

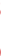

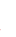
5 6 (4) 0 1 2 3 4 57 58 9 
Table 1 Patients' characteristics and outcome

\begin{tabular}{lcccc}
\hline Characteristics & Age/sex & Chest CT-scan & Hospital course & Outcome \\
\hline Patient 1 & $45 /$ male & Figure 1 & Progression to ARDS & Death \\
Patient 2 & $56 /$ male & Figure 2 & Progression to ARDS & Death \\
Patient 3 & 29/female & Figure 3 & Resolution of symptoms & Discharge to home on day 7 \\
\hline
\end{tabular}

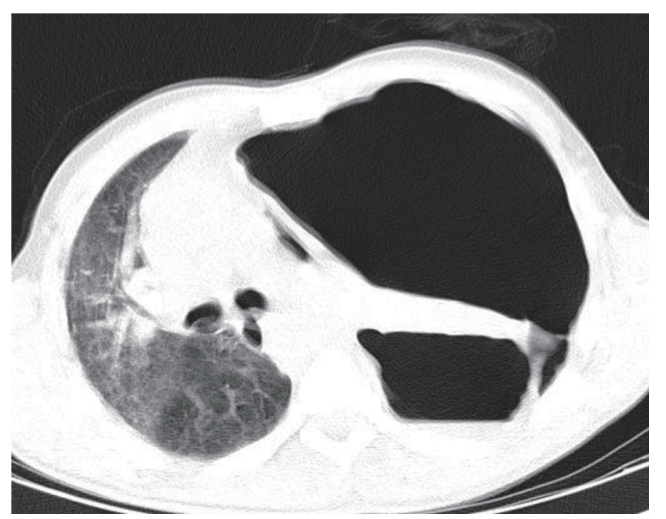

Figure 1 Chest CT scan of patient 1 showing a large left sided pneumothorax.

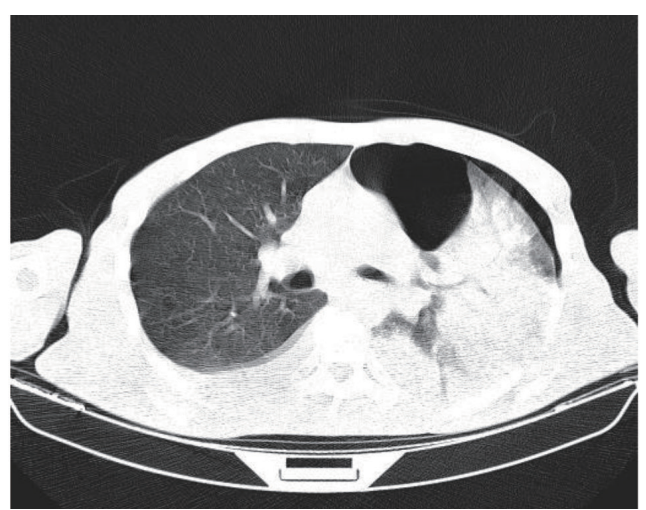

Figure 2 Chest CT scan of patient 2 showing a moderate left sided pneumothorax.

he was febrile (body temperature $38.8^{\circ} \mathrm{C}$ ), tachycardic (heart rate 120 beats per minute), and hypotensive (blood pressure $90 / 70 \mathrm{mmHg}$ ). The acute physiology and chronic health evaluation (II) (APACHE II) score was 12. The ventilator was set on volume assist-control ventilation (ACV) mode with a positive end-expiratory pressure (PEEP) of 18 and a tidal volume (TV) of $6 \mathrm{cc} / \mathrm{kg}$. Despite these measures he remained hypoxemic. Shortly after he progressed to acute respiratory distress syndrome (ARDS) and died 12 hours after arrival to the ICU. The result of his RT-PCR analysis 77 returned positive for COVID-19 three days after his death.

\section{Patient 2}

On March 18, 2020, a 56-year-old man presented to the emergency department with 3-day history of fever, cough, and chest pain. He was a 37 pack-year smoker and otherwise healthy with no recent history of trauma. He reported close contact with a COVID-19 positive patient. During the initial evaluation, he had a body temperature of $37.7^{\circ} \mathrm{C}$, blood pressure of $120 / 80 \mathrm{mmHg}$, pulse rate of 99 beats per minute, respiratory rate of 22 breaths per minute, and his oxygen saturation was $91 \%$ on room air. He had normal mental status with a Glasgow Coma Scale (GCS) of 15 . Lung auscultation revealed decreased breath sounds over the left hemithorax. Initial blood work demonstrated the following: WBC $26,000 \mu \mathrm{L}$ with $6 \%$ lymphocytes; and CRP $61 \mathrm{mg} / \mathrm{L}$. A COVID-19 PCR test was obtained. Chest CT scan showed a moderate size pneumothorax on the left side (Figure 2). A left thoracostomy tube was placed and due to high clinical suspicion, he was admitted to the COVID-19 Unit. His APACHE II score on admission to the unit was 14. On the second day of admission, he became febrile (body temperature $38^{\circ} \mathrm{C}$ ) and hypotensive (blood pressure $80 /$ pulse). His respiratory status rapidly decompensated and he required intubation with mechanical ventilation; he was placed on ACV mode with a PEEP of 17 and TV of $5 \mathrm{cc} / \mathrm{kg}$. Despite all efforts, he died 30 hours after admission to the unit. The result of RT-PCR for COVID-19 returned positive 2 days after patient's death.

\section{Patient 3}

On March 21, 2020, a 29-year-old woman presented to the emergency department with 4-day course of low-grade fever and chest pain. She was otherwise healthy with no recent pregnancy or child delivery, and denied any trauma or contact with confirmed COVID-19 patients. During the initial evaluation, she had a body temperature of $37.6{ }^{\circ} \mathrm{C}$, 
blood pressure of $100 / 70 \mathrm{mmHg}$, pulse rate of 100 beats per minute, respiratory rate of 24 breaths per minute, and oxygen saturation of $91 \%$ on room air. She had a GCS of 15 with an APACHE II score of 3. Lung auscultation revealed decreased breath sounds over the right hemithorax. Initial blood work was a follow: WBC $12,500 \mu \mathrm{L}$ with $16 \%$ lymphocytes; and CRP $7.7 \mathrm{mg} / \mathrm{L}$. A COVID-19 PCR test was obtained. Chest CT scan showed a large pneumothorax on the right side (Figure 3). A thoracostomy tube was placed with resolution of the pneumothorax. She was then admitted to the surgical ward for thoracostomy tube management and supportive care. Her symptoms improved and the thoracostomy tube was removed on hospital day 4; she was discharged home on hospital day 7. Figure $4 A$ shows the chest $\mathrm{X}$-ray after thoracostomy tube placement and

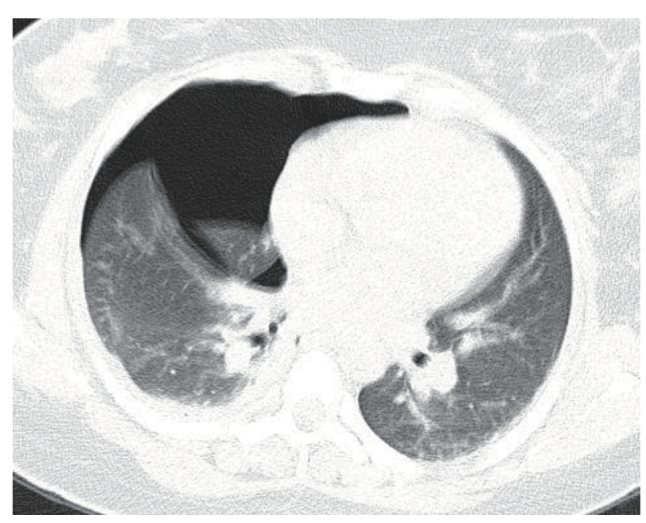

Figure 3 Chest CT scan of patient 3 showing a large right sided pneumothorax.
Figure $4 B$ shows the chest X-ray after removal of the tube. 132 The result of RT-PCR returned positive for COVID-19 133 while patient was still in the hospital.

Pneumothorax has been reported as a finding in viral pneumonia as early as during the $1918 \mathrm{H} 1 \mathrm{~N} 1$ influenza pandemic (3). Studies published about more recent pneumonia pandemics also report development of pneumothorax in patients with severe acute respiratory syndrome (SARS) and also during Middle East respiratory syndrome (MERS) and 2009-2010 H1N1 influenza outbreaks. However, this pattern of pneumothorax almost always developed during mechanical ventilation or late in the course of disease.

In a study of 41 SARS patients on mechanical ventilation, 5 patients $(12 \%)$ were found to develop pneumothorax at a mean of 8 days following initiation of mechanical ventilation. The patients who went on to develop pneumothorax were noted to have a higher respiratory rate on admission, lower $\mathrm{PaO}_{2} / \mathrm{FiO}_{2}$ ratio, and high $\mathrm{PaCO}_{2}$ levels, but did not significantly differ in ventilator pressure and adjusted volumes compared to the patients who did not develop pneumothorax (4). Sihoe and colleagues presented a series of 6 SARS patients with spontaneous pneumothorax, 4 of which developed pneumothorax without prior positive pressure ventilation. None of them had pneumothorax on presentation and developed the condition at a mean 24.3 days (range, 14 to 37 days) following admission (5). Similarly, two retrospective reviews of intubated MERS patients reported development of pneumothorax in $7.1 \%$ and $30 \%$ of their patients $(6,7)$. Also multiple cases of
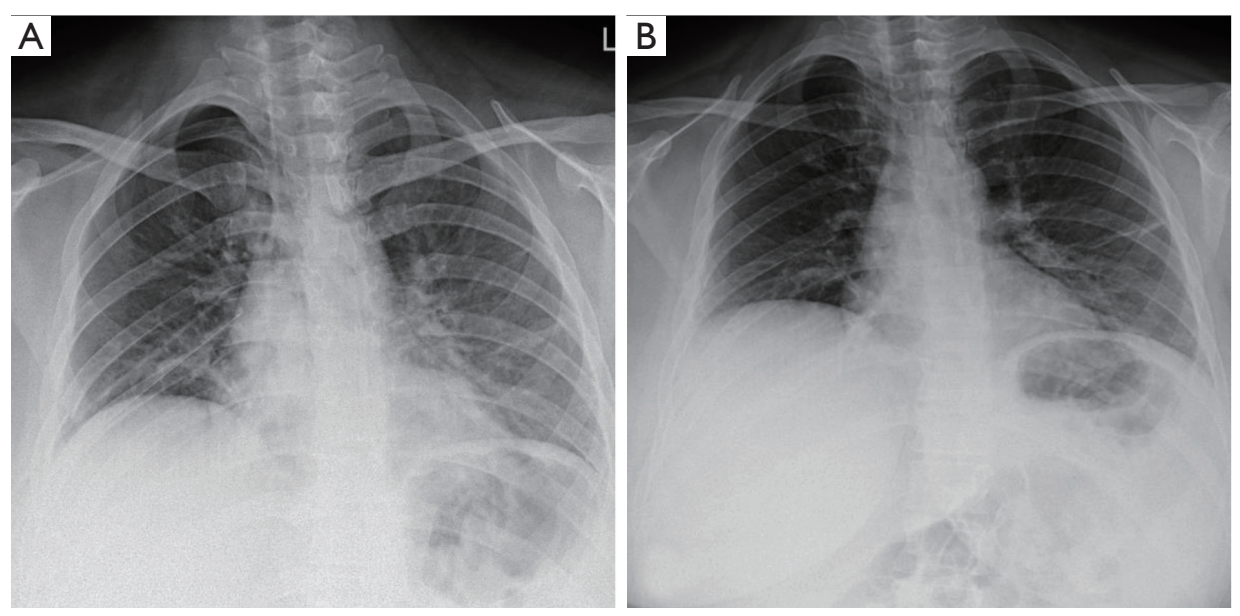

Figure 4 Chest X-ray of patient 3. (A) after thoracostomy tube placement; (B) after removal of the tube and resolution of pneumothorax. 
pneumothorax occurring in $\mathrm{H} 1 \mathrm{~N} 1$ influenza patients have been reported. Guo and colleagues presented a 56-yearold male who was admitted for H1N1 pneumonia, rapidly decompensated with ARDS, developed a pneumothorax shortly after intubation, and eventually died (8). Another report from Turkey described a 31-year-old pregnant female admitted for H1N1 influenza, and who was recovering from ARDS. On admission day 37th, 2 days following extubation, she was found to have a right-sided pneumothorax, which was treated. She then developed a recurrent pneumothorax one week following discharge (9).

The interval of time to progression to severe disease, need for intubation and development of ARDS in our patients was shorter than previous reports for COVID-19 patients. Prior COVID-19 studies have reported that symptoms typically progress over one week prior to admission and dyspnea manifests at 5-8 days after symptom onset (10-12). The 8-day mark is also the median reported timeframe for the development of ARDS (11). Pan et al. also showed that CT findings of COVID-19 appeared to be most severe around this same timeframe (13). The most peculiar feature of the cases presented in this communication is that pneumothorax developed at the time of presentation and within a short time following symptom onset. This is a stark difference from other viral pneumonias in which pneumothorax may occur weeks after presentation and in the setting of progressive ARDS with positive pressure ventilation.

While the chronology differs from the presented COVID-19 patients, experience with SARS suggests that the pathophysiologic changes of viral pneumonia may play a role in the development of pneumothorax independent of ventilator barotrauma. Earlier studies proposed that these pneumothoraxes were resultant from the formation of subpleural tubercles which adhered to the pleura, eventually forming emphysematous bulla (14). Inflammation of small airways increases alveolar pressures causing extravasation of inspired air into the lung hilum and pneumomediastinum. Subsequent rupture of mediastinal parietal pleural then results in air leak into the pleural space and pneumothorax (15). Alternatively, pulmonary necrosis may cause rupture of the alveolus directly into the pleural space, which has been more classically described in pneumocystis pneumonias (15). Predominance of peripheral lung involvement on CT scan of COVID-19 patients may explain increased, earlier inflammation at the pleura, resulting in earlier pneumothorax compared to other viral pneumonias.

On a cellular level, there are also immunological changes which may play a role in potentiation of pneumothorax. 210 Studies have suggested that bulla formation in patients with 211 primary spontaneous pneumothorax may be propagated 212 by inflammatory breakdown of elastic fibers. An immune 213 response, mediated by respiratory epithelium, eosinophils, 214 and innate lymphoid cells (ILCs), has been proposed as 215 an aspect of pathophysiology in primary spontaneous 216 pneumothorax (16). Of these cells the ILC-1 subtype 217 has been show to increase in number in response to 218 intracellular pathogens including viruses (17). Further 219 work is needed to clarify is similar processes are at play 220 during viral pneumonias. A key immunological finding 221 in SARS and MERS patients, was viral interference with 222 innate, interferon (INF) mediated, immune response. It has 223 been suspected that SARS-CoV-2 likely induces a similar 224 modification of the innate immune system, but will require 225 additional clarification (18). This observation may also 226 explain why COVID-19 may present with minimal if any 227 symptoms children, when innate immunity is most robust. 228

Outcomes data has placed focus on identifying 229 COVID-19 patients who are at higher risk for morbidity 230 and mortality. Advanced age, male sex, hypertension, cardiac 231 disease, diabetes, chronic pulmonary disease, chronic kidney 232 disease, malignancy, and immunocompromised state have 233 all been identified as risk factors (11). Several laboratory 234 findings have also been implicated as markers of severe 235 disease including lymphopenia as well as elevated CRP, 236 Ferritin, D-Dimer, and LDH (12). Interestingly, both male 237 patents died in this series and both were noted to have 238 elevated CRP on presentation (Table 2). Other than the 239 fact that patient 2 was an active smoker, the patients were 240 otherwise young without pre-existing comorbidities.

A systematic review of 37 COVID-19 studies establishing a proposed CT scoring system (COVID-RADS), classified pneumothorax as an atypical (grade 1) CT finding of COVID-19 with a low-level suspicion $(19,20)$. The COVID-19 patients in the current case series had unusual presenting radiographic findings. This unusual presentation however was associated with devastating outcomes. The most concerning feature of these scenarios is that pneumothorax developed within a short time following symptom onset. This is a stark difference from other viral pneumonias in which pneumothorax may occur weeks after presentation and in the settings of progressive ARDS and positive pressure ventilation. All of the presented patients came to the emergency room with typical respiratory viral complaints, and pneumothorax was unlikely in a typical, initial differential diagnosis. Due to institutional 
Table 2 Initial laboratory findings of the patients

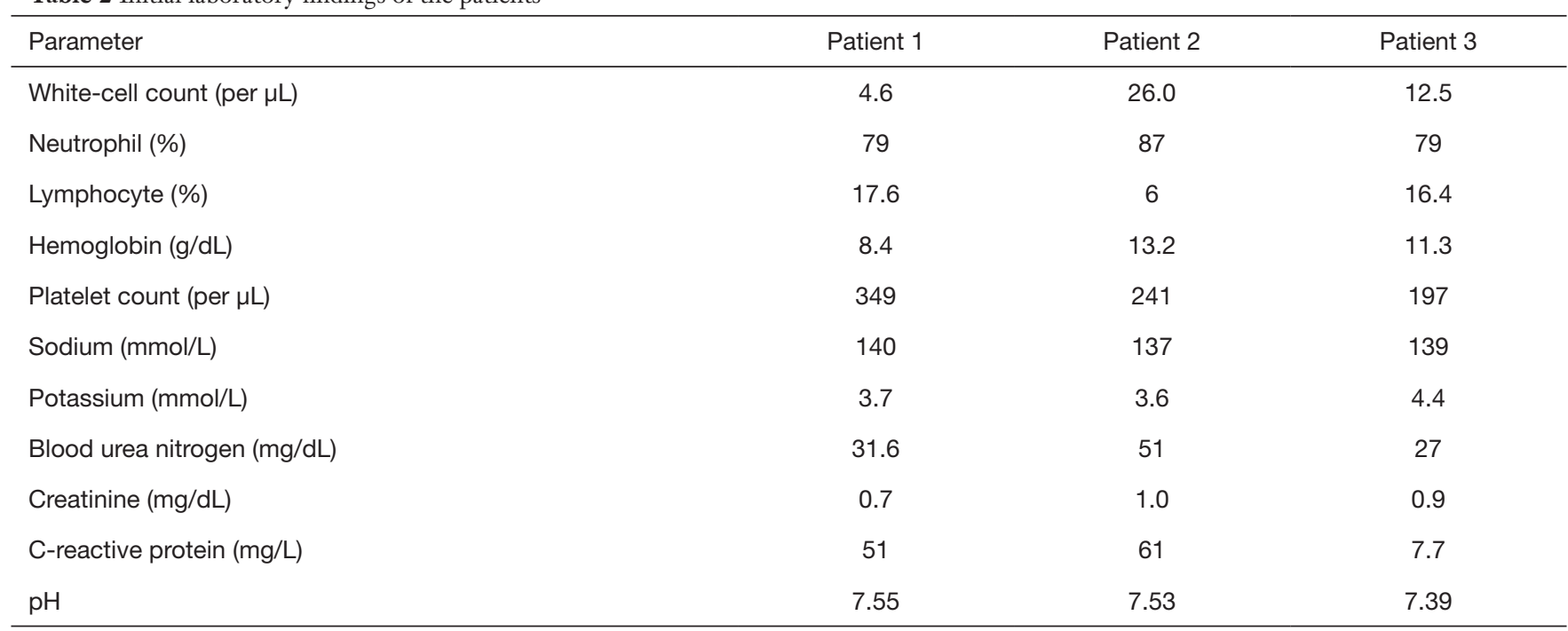

Acknowledgments

Funding: None.

Footnote

Provenance and Peer Review: This article was a free submission to the journal. The article was sent for external peer review. atypical presentations.

It should be emphasized that a causal relationship between COVID-19 and pneumothorax cannot be concluded from this series and theses uncommon presentations may be confounded by unknown patient and regional variables. The presence of prior bullous disease, underlying connective tissue disease, hormonal irregularities, environmental exposure, and vigorousness of coughing are unknown considerations. An additional thought is that the clinical course may not reflect the actual exposure-onset timeline. Reports from China have suggested that the clinical and radiographic disease severity rapidly worsens around one week after initial onset of symptoms in severe COVID-19 cases (11-13), with ARDS and radiographic severity progressing over one week These patients may have experienced a relatively asymptomatic early disease course and presented during a later disease process. Even considering these factors, three COVID-19 patients having pneumothorax on presentation remains a striking entity.
Conflicts of Interest: All authors have completed the ICMJE uniform disclosure form (available at http://dx.doi. org/10.21037/jtd-20-1687). The authors have no conflicts of interest to declare.

Ethical Statement: The authors are accountable for all aspects of the work in ensuring that questions related to the accuracy or integrity of any part of the work are appropriately investigated and resolved. In addition, based on our institution policy, all the patients who present to our information for clinical research purposes.

Open Access Statement: This is an Open Access article distributed in accordance with the Creative Commons Attribution-NonCommercial-NoDerivs 4.0 International License (CC BY-NC-ND 4.0), which permits the nonhospital, give consent for using their de-identified clinical 
commercial replication and distribution of the article with the strict proviso that no changes or edits are made and the original work is properly cited (including links to both the formal publication through the relevant DOI and the license). See: https://creativecommons.org/licenses/by-nc-nd/4.0/.

\section{References}

1. World Health Organization. Coronavirus disease 2019 (COVID-19): situation report. Available online: https:// apps.who.int/iris/bitstream/handle/10665/331613/ nCoVsitrep27Mar2020-eng.pdf

2. Reuters "Iran Confirms 13 More Coronavirus Cases, Two Deaths - Health Ministry." Reuters, Thomson Reuters, 21 Feb 2020, Available online: https://reuters.com/article/ china-health-iran/iran-confirms-13-more-coronaviruscases-two-deaths-health-ministry-idUSD5N27F006

3. Neffson AH, Bullowa JGM. Influenza with Simultaneous Bilateral Spontaneous Pneumothorax and Subcutaneous Emphysema: Report of a Case, with Comment on The Mechanism of Production. Arch Otolaryngol 1938;28:388-98.

4. Kao HK, Wang JH, Sung CS, et al. Pneumothorax and mortality in the mechanically ventilated SARS patients: a prospective clinical study. Crit Care 2005;9:R440-5.

5. Sihoe AD, Wong RH, Lee AT, et al. Severe acute respiratory syndrome complicated by spontaneous pneumothorax. Chest 2004;125:2345-51.

6. Saad M, Omrani AS, Baig K, et al. Clinical aspects and outcomes of 70 patients with Middle East respiratory syndrome coronavirus infection: a single-center experience in Saudi Arabia. Int J Infect Dis 2014;29:301-6.

7. Das KM, Lee EY, Al Jawder SE, et al. Acute Middle East Respiratory Syndrome Coronavirus: Temporal Lung Changes Observed on the Chest Radiographs of 55 Patients. AJR Am J Roentgenol 2015;205:W267-74.

8. Guo HH, Sweeney RT, Regula D, et al. Best cases from the AFIP: fatal 2009 influenza A (H1N1) infection, complicated by acute respiratory distress syndrome and pulmonary interstitial emphysema. Radiographics 2010;30:327-33.

9. Bor C, Demiră̆ K, Uyar M, et al. Recurrent Spontaneous
Pneumothorax during the Recovery Phase of ARDS Due to H1N1 Infection. Balkan Med J 2013;30:123-5.

10. Guan WJ, Ni ZY, Hu Y, et al. Clinical Characteristics of Coronavirus Disease 2019 in China. N Engl J Med 2020;382:1708-20.

11. Wang D, Hu B, Hu C, et al. Clinical Characteristics of 138 Hospitalized Patients With 2019 Novel CoronavirusInfected Pneumonia in Wuhan, China. JAMA 2020;323:1061-9.

12. Huang C, Wang Y, Li X, et al. Clinical features of patients infected with 2019 novel coronavirus in Wuhan, China. Lancet 2020;395:497-506.

13. Pan F, Ye T, Sun P, et al. Time Course of Lung Changes at Chest CT during Recovery from Coronavirus Disease 2019 (COVID-19). Radiology 2020;295:715-21.

14. Spontaneous Pneumothorax. Available online: https:// journal.chestnet.org/article/S0096-0217(16)30407-1/ fulltext

15. Sahn SA, Heffner JE. Spontaneous pneumothorax. N Engl J Med 2000;342:868-74.

16. Kwon BI, Hong S, Shin K, et al. Innate type 2 immunity is associated with eosinophilic pleural effusion in primary spontaneous pneumothorax. Am J Respir Crit Care Med 2013;188:577-85.

17. Bernink JH, Peters CP, Munneke M, et al. Human type 1 innate lymphoid cells accumulate in inflamed mucosal tissues. Nat Immunol 2013;14:221-9.

18. Prompetchara E, Ketloy C, Palaga T. Immune responses in COVID-19 and potential vaccines: Lessons learned from SARS and MERS epidemic. Asian Pac J Allergy Immunol 2020;38:1-9.

19. Salehi S, Abedi A, Balakrishnan S, et al. Coronavirus disease 2019 (COVID-19) imaging reporting and data system (COVID-RADS) and common lexicon: a proposal based on the imaging data of 37 studies. Eur Radiol 2020;30:4930-42.

20. Salehi S, Abedi A, Balakrishnan S, et al. Coronavirus Disease 2019 (COVID-19): A Systematic Review of Imaging Findings in 919 Patients. AJR Am J Roentgenol 2020;215:87-93.
Cite this article as: Ayazi S, Zebarjadi J, Grubic AD, Tahmasbi H, Ayazi K, Jobe BA. Pneumothorax as the presenting manifestation of COVID-19. J Thorac Dis 2020;12(12):74887493. doi: $10.21037 /$ jtd-20-1687 